\title{
Small Cell Neuroendocrine Carcinoma of the Urinary Bladder: Rare Entity Associated to Poor Prognosis
}

\author{
M Chraibi ${ }^{1}$ and $M$ Barqui $^{2}$ \\ ${ }^{1}$ Pathology Department, Centre Hospitalo-Universitaire Mohammed VI Marrakech, Oujda, Morocco \\ ${ }^{2}$ Urology Department, Centre Hospitalo-Universitaire Mohammed VI Marrakech, Oujda, Morocco
}

*Corresponding author: M Chraïbi, Pathology Department, Mohamed VI CHU, Oujda, Morocco, Tel: 00212-6-41-13-56-76

\begin{abstract}
Neuroendocrine Small cell Carcinoma (SCC) of the urinary bladder is a rare tumor. Pure Small Cell Carcinoma is uncounted from 0.5 to $1 \%$ of all primitive urinary tumors. The diagnosis is based on transurethral resection. The old age, and smoking are putative risk factors. A genetic predisposition is unknown.

Pure SCC of the bladder display metastasis and lymph nodes. Viscera and vertebral bones are the most common sites of the metastasis. Painless gross hematuria is the most common clinical feature. The prognosis is very poor. The overall survival rate at five years, in advanced stages does not exceed $8 \%$.

SCC of the urinary bladder can be treated with local resection of the tumor or radical cystectomy with neoadjuvant or adjuvant chemotherapy. Radiation therapy is used as an alternative to radical cystectomy or as a palliative measure.
\end{abstract}

\section{Introduction}

Small Cell Carcinoma of the bladder is a rare, aggressive, poorly differentiated neuroendocrine neoplasm [1,2]. Since the tumor is very rare, the pathogenesis is uncertain. Some authors proposed a urothelial cell origin, but others defend a stem cell theory. Recently, molecular studies have indicated that the small cell carcinoma and the urothelial carcinoma are derived from the same clonal population $[1,3]$. We report a case of small cell carcinoma of urinary bladder, initially reported as poorly differentiated urothelial carcinoma on transurethral resection. We discuss here the clinical and the morphological features of this entity.

\section{Case Report}

A 59-year-old man presented with hematuria, particularly at the end of the micturition. The patient was a builder, a chronic smoker and alcoholic. His past and family history was insignificant.

A pelvic Ultrasound revealed a large mass measuring $7 \times 5 \mathrm{~cm}$, from lateral right wall of the urinary bladder with no evidence of calculi. Contrast-enhanced computed Tomography Scan confirmed the anatomical location of the mass along with involvement of the adjacent musculature (Figure 1).

Patient underwent cystoscopy with biopsy of mass. The first histopathological examination reported a poorly differentiated urothelial carcinoma.

Radical cystoprostatectomy with pelvic lymphadenectomy after chemotherapy was carried out and the specimen was sent to the Department of Pathology for histopathological analysis.

\section{Pathological Examination}

The composite specimen of urinary bladder, prostate with seminal vesicles, both sided ureters and perivesical adipose tissue measured $10 \times 8 \times 5.5 \mathrm{~cm}$. On cutting, the urinary bladder showed a mass measuring $5 \mathrm{~cm}$ in its greatest dimensions (Figure 2). Sections from the representative areas on microscopy showed sheets and nests of loosely cohesive, small round to oval cells with hyperchromatic coarsely granular nuclear chromatin and scant cytoplasm (Figure 3).

Mitotic figures and nerve invasion were frequent. Tumor invaded complete wall of urinary bladder, the prostatic parenchyma and the seminal vesicles. The neoplastic cells on immunohistochemistry exhibited Chromogranin A and focal cytokeratin positivity and 


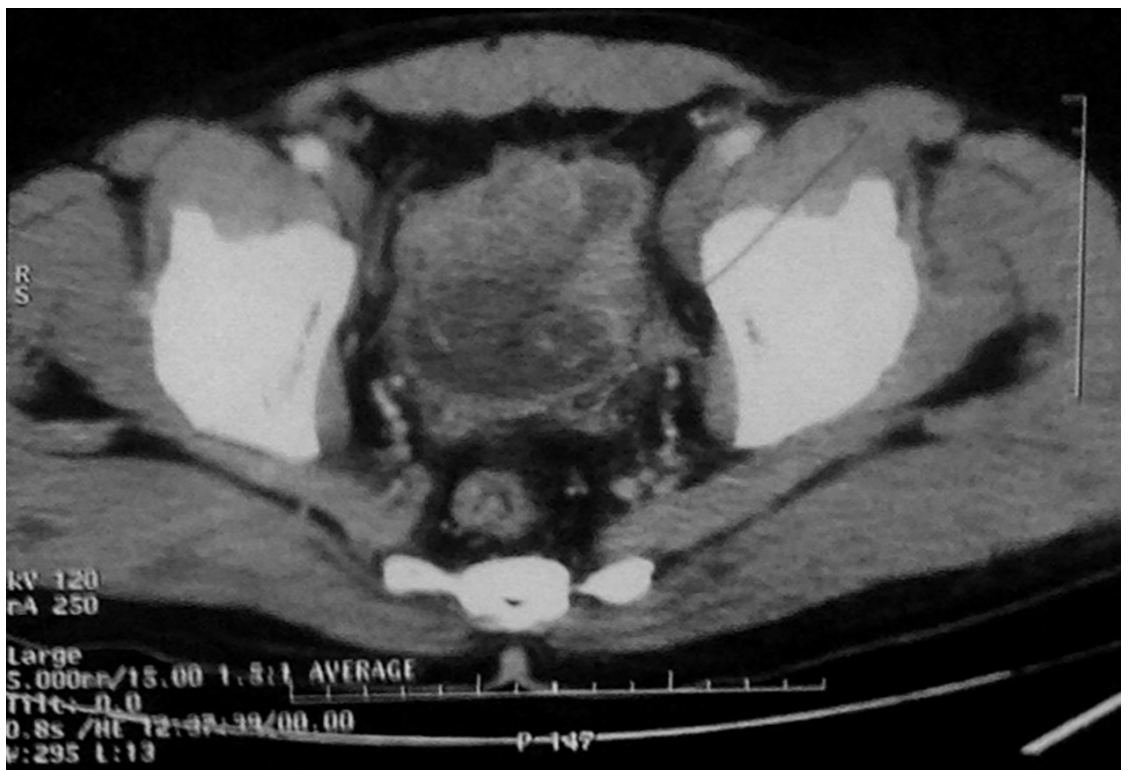

Figure 1: Contrast-enhanced CT scan showing mass arising from right wall of the Urinary Bladder (UB).

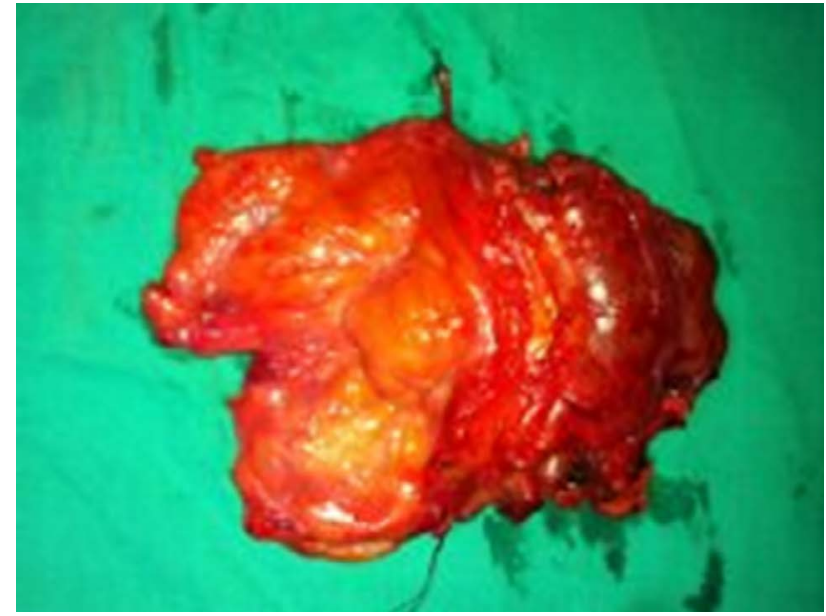

Figure 2: Gross examination: Tumor measured $10 \times 8 \times 5.5$ $\mathrm{cm}$, invades urinary bladder and parenchyma of prostate.

were negative for synaptophysin and CD56.

After the histopathological diagnosis of the small cell carcinoma urinary bladder, patient was advised four cycles of chemotherapy with Cisplatine and Etoposide. Eight months later he deads.

\section{Discussion}

Small Cell Carcinoma (SCC) occurs in the tracheobronchial tree. The extrapulmonary SCC has been described in a variety of organs, including the esophagus, the stomach, the pancreas, the gallbladder, the uterine cervix, the kidney, the urinary bladder, and the prostate. Pure SCC account for only $0.48 \%-1 \%$ of all bladder carcinomas. The clinical manifestation is very similar to other bladder tumors with painless gross hematuria and dysuria being the most frequent signs of the disease. The tumors can also be found during abdominal ultrasound examination. Macroscopically they are usually $4-10 \mathrm{~cm}$ in size and polyp-shaped with ulceration

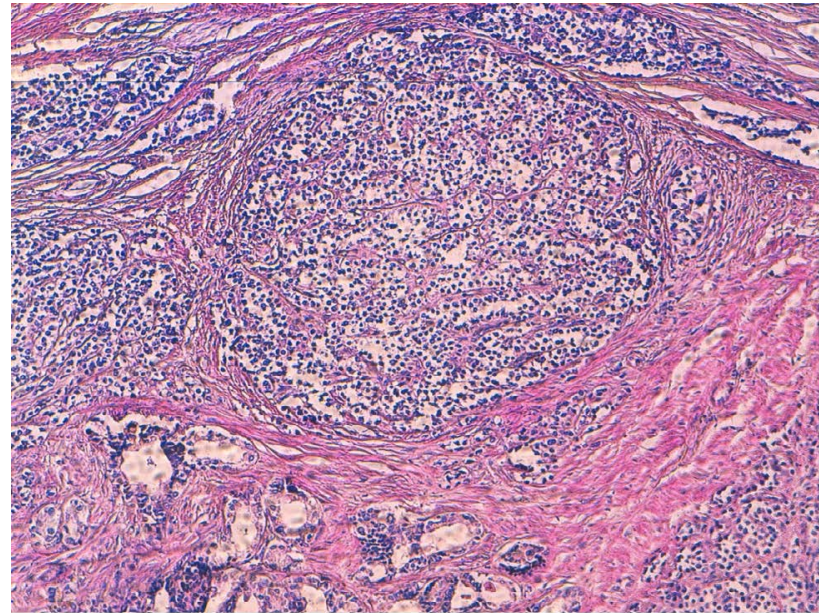

Figure 3: HES. Sheets and nests of loosely cohesive, small round to oval cells with Scant cytoplasm.

in some cases [4]. These features were present in our case. Cigarette smoking, bladder calculi and long-term cystitis are thought to be the etiological factors involved in its pathogenesis $[5,6]$.

The origin of the SCC is still subject of debate, but it is now believed that the SCC of the bladder or the UUT, originates from the totipotent stem cells present in the submucosa of the urinary tract [7].

Histologically, in $68 \%$ of cases, the SCC coexists with classic the urothelial carcinomas or adenocarcinomas of the bladder. Our case was diagnosed as pure SCC. Microscopic examination reveals a tumor composed of sheets and nests of loosely cohesive, small, round, or oval cells with very scanty cytoplasm. The tumor cell nuclei are hyperchromatic with coarsely granular chromatin. Nuclear molding can be seen. Mitotic figures are present and may be frequent. Nucleoli are absent or small. Tumor necrosis is often present. The urinary cytology shows often single and loosely cohesive 
clusters of tumor cells with typical SCC morphology. Neuroendocrine markers, such as chromogranin A, synaptophysin, CD56, and neuron specific enolase (NSE), are often focally or diffusely positive for these tumors by immunohistochemical methods, and they are useful tools that aid in establishing the diagnosis. A cocktail of cytokeratin (CK) markers is often nonreactive, but low molecular cytokeratin, CAM5-2, and epithelial membrane antigen (EMA) are mostly positive [4].

Unfortunately, the optimal management is not well defined. Therapeutic modalities vary and include transurethral resection, cystectomy, radiation therapy and systemic chemotherapy [8]. Surgical resection (radical cystectomy and extended pelvic lymphadenectomy) alone is unlikely to be curative, unless the tumor is confined to the bladder. Combination therapy with adjuvant or neoadjuvant chemotherapy is recommended.

Prognosis of SCC remains poor due to a very aggressive behavior with up to $25 \%$ of patients presenting metastatic disease at the time of diagnosis and absence of symptoms at the beginning of disease. The overall 5-year survival rate for patients with SCC, is variable and ranges from $8 \%$ to $25 \%$ [4].

\section{Conclusion}

Primitive Pure Neuroendocrine small cell carcinoma of urinary bladder is exceptional. It is associated to poor prognosis. The diagnosis is based on morphological examination and immunochemistry. Further studies are necessary to define the novel therapies, which are needed to improve outcomes.

\section{References}

1. Cheng L, Jones TD, McCarthy RP, Eble JN, Wang M, et al. (2005) Molecular genetic evidence for a common clonal origin of urinary bladder small cell carcinoma and coexisting urothelial carcinoma. Am J Pathol 166: 1533-1539.

2. Trias I, Algaba F, Condom E, Español I, Seguí J, et al. (2001) Small cell carcinoma of the urinary bladder. Presentation of 23 cases and review of 134 published cases. Eur Urol 39: 85-90.

3. Shahab N (2007) Extrapulmonary small cell carcinoma of the bladder. Semin Oncol 34: 15-21.

4. Radosław Starownik, Agnieszka Korolczuk, Krzysztof Bar, Paweł Płaza, Jacek Kiś, et al. (2013) Partial cystectomy in a 76 year old patient suffering from small cell carcinoma of the urinary bladder. Cent European J Urol 66: 159-162.

5. Cheng L, Pan CX, Yang XJ, Lopez-Beltran A, MacLennan GT, et al. (2004) Small cell carcinoma of the urinary bladder: A clinicopathologic analysis of 64 patients. Cancer 101: 957-962.

6. Sved P, Gomez P, Manoharan M, Civantos F, Soloway MS (2004) Small cell carcinoma of the urinary bladder. BJU Int 94: 12-17.

7. Naturale RT, Maclennan GT (2006) Small cell carcinoma of the bladder. J Urol 176: 781.

8. Dahm P, Gswhwend JE (2003) Malignant non-urothelial neoplasm of the urinary bladder: A review. Eur Urol 44: 672-681. 